\title{
Tendencias de Investigación en la Cadena de Suministro de Residuos Sólidos Municipales
}

Jenny F. Gaviria-Cuevas(1), Jonathan Soto-Paz ${ }^{(2)}$, Pablo C. Manyoma-Velasquez ${ }^{(1)}$ y Patricia Torres-Lozada ${ }^{(2)}$ (1) Univ. del Valle, Facultad de Ingeniería, Grupo de Investigación Logística y Producción - LOGYPRO, Calle 13 No. 100-00, Cali-Colombia; (e-mail: jenny.gaviria@correounivalle.edu.co; jonathan.soto.paz@correounivalle.edu.co) (2) Univ. del Valle, Facultad de Ingeniería, Grupo de Investigación Estudio y Control de la Contaminación AmbientalECCA, Calle 13 No.100-00, Cali-Colombia. (e-mail: pablo.manyoma@correounivalle.edu.co; patricia.torres@correounivalle.edu.co)

Recibido Oct. 3, 2018; Aceptado Dic. 13, 2018; Versión final Feb. 2, 2019, Publicado Ago. 2019

\section{Resumen}

El propósito de este trabajo es identificar las tendencias en investigación a nivel global en la cadena de suministro de la Gestión Integral y Sostenible de Residuos Sólidos (GISRS), en cuanto a la gestión y tratamiento de los Residuos Sólidos Municipales (RSM). Para este fin, se realizó una búsqueda exhaustiva de artículos en bases de datos especializadas, información que se procesó en Bibexcel, Pajek, VoSviewer@ y Tagxedo, software libre para análisis bibliométrico. Como resultado, se encontró el predominante uso de tecnologías de tratamiento de los RSM con fines de recuperación energética. Por otra parte, la GISRS, analizada como una cadena de suministro, involucra decisiones de tratamiento de RSM, actores involucrados y aspectos ambientales, técnicos, sociales y económicos. Finalmente, se identificaron cuatro grandes brechas u oportunidades de investigación que pueden ser afrontadas con herramientas de la Ingeniería para soporte a decisiones que involucren flujos de recursos, dinero y energía.

Palabras clave: residuos sólidos municipales; tratamiento de residuos; sostenibilidad; cadenas de suministro

\section{Trends in Research on the Supply Chain Management of Municipal Solid Waste}

\begin{abstract}
The purpose of this paper is to identify trends in research at a global level in the supply chain of the Integral and Sustainable Management of Solid Waste (ISMSW), in terms of management and treatment of Municipal Solid Waste (MSW). For this purpose, an exhaustive search of articles in specialized databases was carried out, information that was processed using the free software applications Bibexcel, Pajek, VoSviewer @ and Tagxedo for bibliometric analysis. As a result, it was found a predominant use of technologies to treat MSW for the purpose of energy recovery. On the other hand, the ISMSW analyzed as a supply chain, involves SWM treatment decisions, actors involved and environmental, technical, social and economic aspects. Finally, four major research gaps or opportunities were identified that can be addressed with engineering tools to support decisions that involve resources, money and energy flows.
\end{abstract}

Keywords: solid waste management; solid waste treatment; sustainability; supply chain 


\section{INTRODUCCIÓN}

De acuerdo con las estadísticas globales, diez años atrás se generaban $0.64 \mathrm{Kg} /$ per cápita/día de Residuos Sólidos Municipales - RSM, equivalentes a 0.68 billones de toneladas por año; actualmente se generan 1.2 $\mathrm{Kg} /$ per cápita/día, equivalentes a 1.3 billones de toneladas por año. Para el año 2025, estas cifras pueden incrementarse a $1.42 \mathrm{Kg} / \mathrm{per}$ cápita/día (2.2 billones de toneladas por año) (Banco Mundial, 2012). Debido a la creciente generación de RSM a nivel mundial, se necesita una gestión adecuada para dichos residuos, teniendo en cuenta aspectos económicos, técnicos, ambientales y sociales, lo que redunda en una mayor sostenibilidad, que se refiere a "la satisfacción de las necesidades actuales sin comprometer la capacidad de la generaciones futuras para satisfacer las suyas, garantizando el equilibrio entre crecimiento económico, cuidado del medio ambiente y bienestar social" (OXFAM Intermón, 2018). La Gestión Integral y Sostenible de los Residuos Sólidos (GISRS) es un proceso que involucra diversas tecnologías y disciplinas asociadas con el control de la generación, separación la fuente, almacenamiento, presentación, recolección, transporte y transferencia, tratamiento, aprovechamiento y disposición final de los residuos sólidos e incluye diferentes actores como grupos involucrados en la gestión de los residuos (Calva-Alejo y Rojas-Caldelas, 2014), así como aspectos ambientales, sociales, financieros y económicos (Klunder y Anschütz, 2001). De acuerdo con autores como Tchobanoglous y Kreith, (2002), el modelo conceptual de la GISRS se asemeja a una cadena de suministro habitual del ámbito industrial, dado que considera flujos de recursos, dinero y energía, tal como se observa en la Fig. 1.

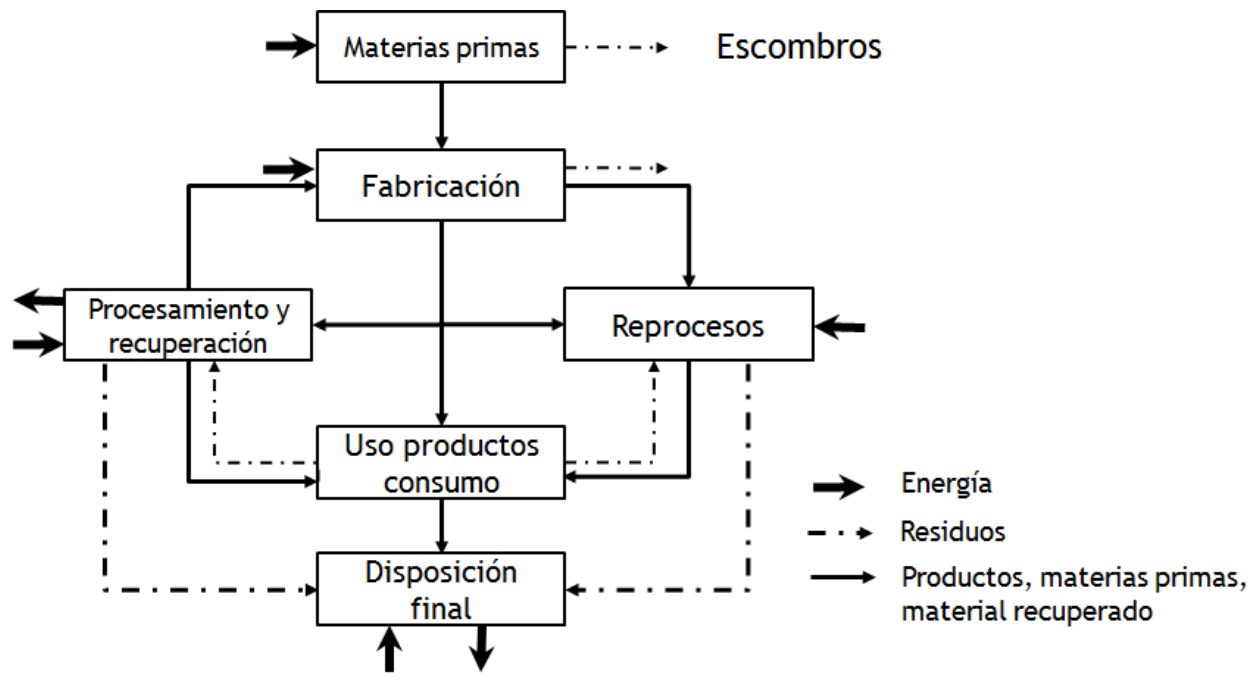

Fig. 1: Cadena de suministro de la GIRS. (Adaptada de Tchobanoglous y Kreith, 2002)

La integración de los eslabones de la cadena de suministro de los RSM en favor del desarrollo sostenible es un reto, especialmente, en países en desarrollo tal como se muestra en ejemplos aplicados como es el caso del estudio de sostenibilidad integral en la cadena de suministro de la producción de biodiesel a base de aceite de palma en algunas regiones de Colombia (Enrique et. al., 2015). En este sentido, la literatura científicotécnica de las diversas áreas de conocimiento ha experimentado un fuerte crecimiento y una dinámica de poder acceder a estadísticas globales en cuanto al desempeño del proceso científico-investigativo. En este sentido, los procesos de vigilancia tecnológica son parte del sistema de gestión de investigación, desarrollo e innovación y utilizan varios enfoques de investigación existente para recolectar la información (López et al, 2016). Uno de estos enfoques es la bibliometría, que como subdisciplina de la cienciometría, proporciona información de los resultados de investigación (Escorcia, 2008). Esta herramienta se utilizó en el presente estudio para determinar y analizar las tendencias en materia de investigación de las cadenas de suministro de la GISRS a nivel global.

\section{MATERIALES Y MÉTODOS}

La metodología inicia definiendo un horizonte de tiempo para realizar la búsqueda de información en el período comprendido entre 2008 y 2018, es decir 10 años de publicaciones de acuerdo a la metodología de Huang et. al., (2011).

Posteriormente se definieron las palabras clave "gestión de los residuos sólidos" y "cadena suministro de los residuos sólidos" términos que se consolidaron en la siguiente ecuación de búsqueda: "gestión de los residuos sólidos" AND "cadena suministro de los residuos sólidos". Con respecto a esta búsqueda se obtuvieron más de 15.000 artículos en las bases de datos consultadas. A este número de artículos se les aplicaron diferentes 
filtros ofrecidos por las bases de datos como "área de interés", "tipo de documentos", "lenguaje", entre otros; hasta que en total por ambas bases de datos (SCOPUS y Web of Science - WoS) se obtuvieron 298 artículos que se procesaron de la siguiente manera: $i$. en Bibexcel desarrollado por Persson, (2017) se analizaron 179 artículos procedentes de la búsqueda en SCOPUS y 119 de la búsqueda en WoS; ii. posteriormente se graficaron los archivos de mapas en Pajeck ${ }^{\circledR}$ como lo aplicaron García et.al., (2015), donde se observan las principales agrupaciones o clúster que se conforman a partir de la co-ocurrencia de términos; iii. estos resultados se procesaron posteriormente mediante el software VOSviewer@) de Leiden University (van Eck y Waltman, 2009) donde se identificaron agrupaciones de términos comunes. Con base en esta búsqueda, se determinaron indicadores bibliométricos como: $i$. nacionalidad de autores y coautores, agrupados por temáticas comunes; ii. países que presentan coautoría por temas comunes; iii. revistas más citadas y relacionadas entre sí. Con el fin de identificar principales áreas de trabajo, inicialmente, se seleccionaron 50 documentos con más de 20 citaciones, los cuales fueron de lectura obligatoria para extraer $i$. las etapas de la GISRS con mayor relevancia en la literatura y ii. número de autores que han investigado sobre temáticas asociadas a diferentes tipos de aprovechamiento de los RSM.

Finalmente se determinan los indicadores bibliométricos como son los Grupos de países con coautorías, Grupos de autores por temáticas y número de documentos asociados, Grupos de revistas por citación y nivel de relación con otras revistas relacionadas y Prioridades de la cadena de suministro de la GISRS, para luego determinar los grupos de brechas u oportunidades de investigación que fueron el resultado de un análisis de co-ocurrencias, basado en las palabras clave que los autores colocaron en sus documentos. Cada brecha se define y se relaciona con herramientas de la ingeniería que pueden ofrecer opciones de solución a dichos temas de profundización.

\section{RESULTADOS}

Como resultado de las búsquedas en las bases de datos, se obtuvieron 298 documentos, que se exportaron a Bibexcel para analizar co-ocurrencias entre sus palabras clave, procedentes tanto del resumen, título y palabras clave de autor(es). Bibexcel genera tres archivos (".net", ".clu" y ".vec") que contienen la información de co-ocurrencias y que fueron procesados en Pajek para obtener una primera red con 4 agrupaciones de términos comunes, las cuales fueron exportadas a VOSviewer@ y Tagxedo. Estas herramientas informáticas permiten identificar cuáles términos son los más comunes en el procesamiento de las búsquedas. En la Fig. 2 se relacionan las palabras clave procedentes de la búsqueda.



Fig. 2: Agrupaciones de los principales tópicos de investigación en la GISRS

La información consignada en la Figura 3, está basada en datos bibliométricos procesados en el software Vosviewer y presenta las coautorías entre los investigadores de la literatura consultada a través de grupos de nacionalidades que posiblemente comparten tópicos de investigación, igualmente, mediante el tamaño de las barras se especifican el número de documentos asociados por el autor principal de cada país, de acuerdo con los parámetros de búsqueda del presente documento. De acuerdo con la literatura consultada, a nivel global los países más productivos son Estados Unidos, Brasil, Reino Unido y por último China, resultados que pueden ser comparables con lo analizado por Chen et. al., (2017) en su trabajo de investigación. 


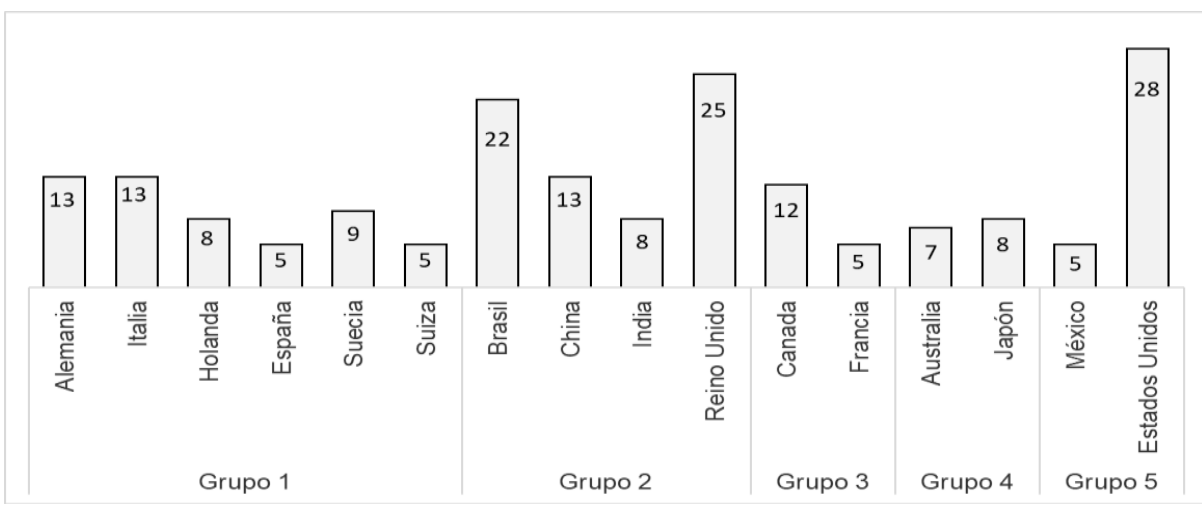

Fig. 3: Grupos de países en coautoría

Otro de los indicadores corresponde a los principales autores que se encontraron en la literatura agrupados por temáticas comunes y con el número de documentos asociados a ellos (Fig. 4); aquí también se especifica el año de mayor publicación de cada uno. Igualmente, las revistas se agruparon por temas de investigación afines, lo que se evidencia en la Fig. 5. El número de conexiones por afinidad temática de cada revista se observa en la parte de color gris claro de cada barra y, en la misma figura se especifican el número de citaciones que tiene cada revista en la parte de color gris oscuro de cada barra. Al lgual que lo encontrado por Fu et. al, (2010) el journal con el mayor número de publicaciones es "Waste Management", que coincide con los resultados de la Fig. 5.

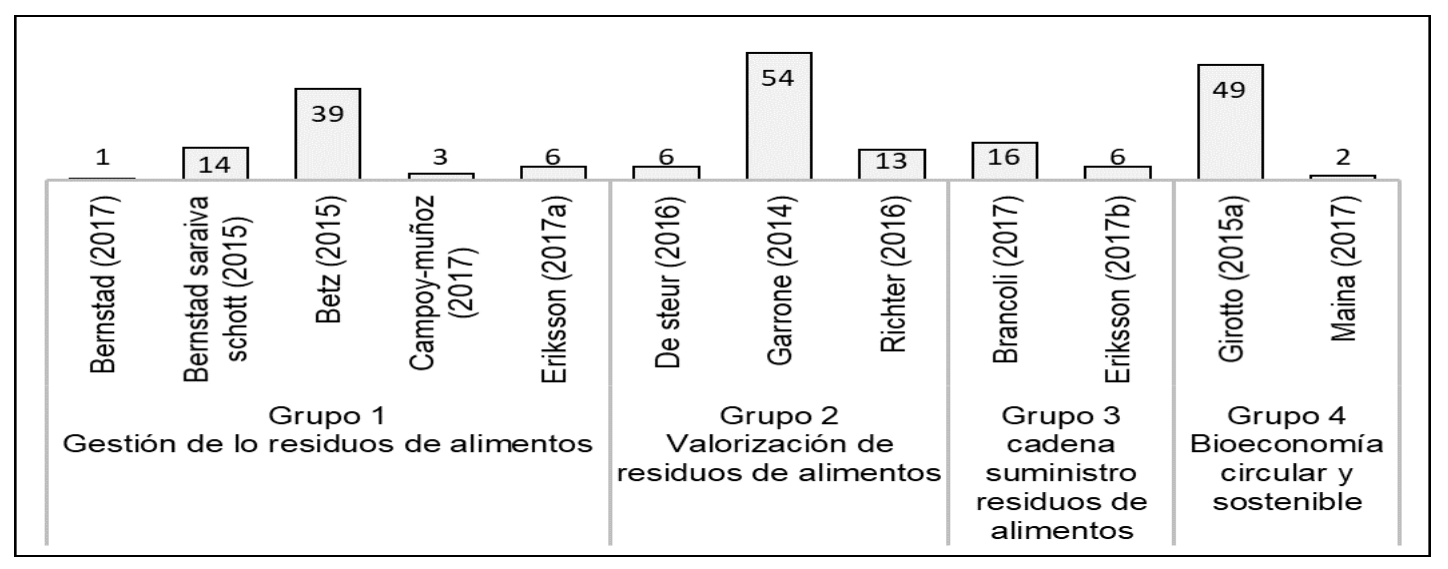

Fig. 4: Grupos de autores por temáticas y número de documentos asociados.

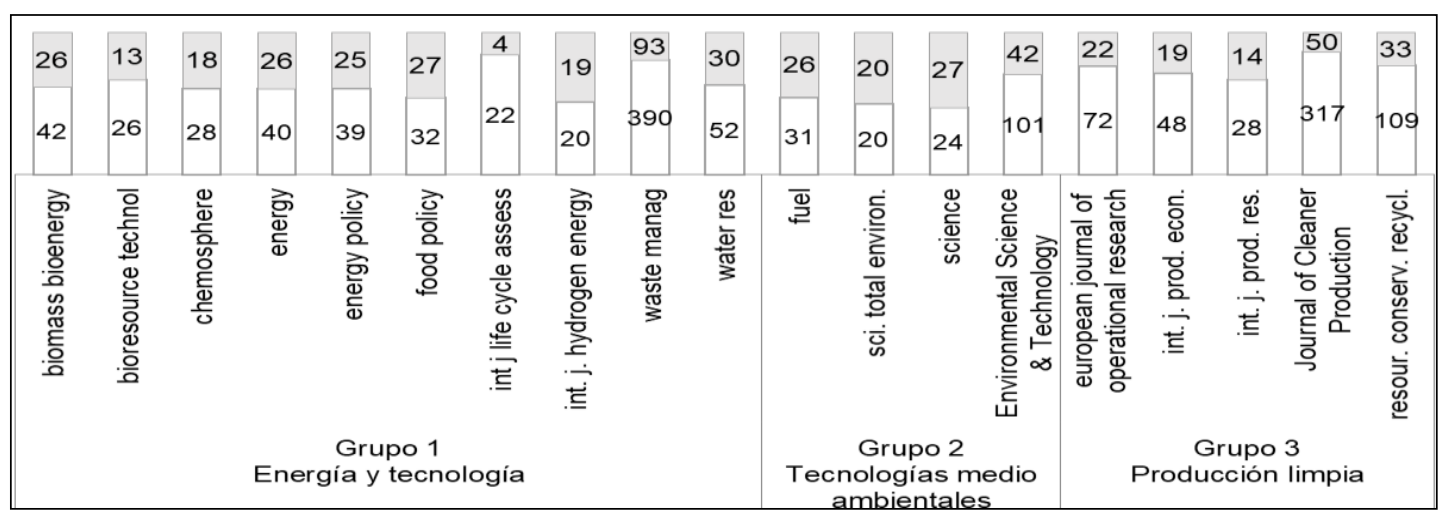

Fig.5: Grupos de revistas por citación y nivel de relación con otras revistas relacionadas.

Los 50 documentos leídos se clasificaron de acuerdo con las fases del sistema de gestión de residuos que corresponden a la generación, recolección y transporte, tratamiento, aprovechamiento y valorización y, finalmente reciclaje, re-utilización y disposición final; de acuerdo a esta categorización se infiere que la fase de tratamiento o aprovechamiento con recuperación energética, seguida por el uso de tecnología más limpia para la prevención en la generación de residuos sólidos representan las prioridades de la cadena de suministro de la GISRS tal como se muestra en la Fig. 6. 


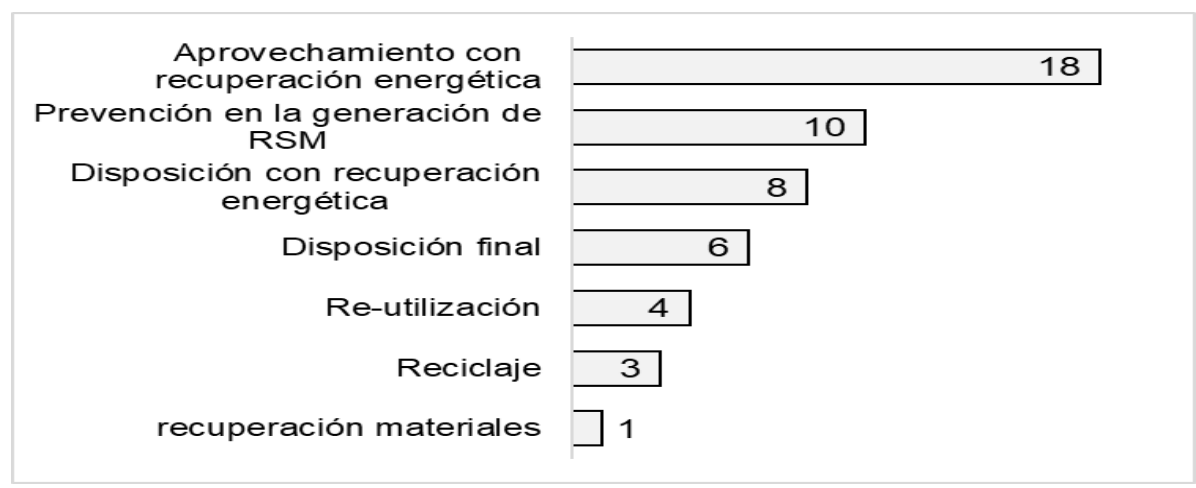

Fig. 6: Prioridades de la cadena de suministro de la GISRS.

En lo referente al aprovechamiento con recuperación de energía, se destacan los tratamiento mecánico, térmico y biológico, lo que promueve la adopción de tecnologías recuperación energética a partir de residuos (WTE) en especial para la valorización de residuos de alimentos, esta inferencia obedece al análisis de trabajos de investigación encontrados en la literatura (Bing et al., 2016; Brancoli et al., 2017; Hupponen et. al., 2015). Se destaca que las cadena de suministro de los residuos de alimentos y la producción más limpia junto con las acciones de mejoramiento en estas cadenas, constituyen ejes fuertes de investigación a nivel global, tal como se encuentra en la literatura (Fehr y Arantes, 2015; Meadows et. al., 2014). Lo anterior conlleva a la identificación de temáticas que pueden ser profundizadas en trabajos o proyectos futuros, razón por la cual, mediante VOSviewer@ se extrajeron palabras clave procedentes de los títulos y resúmenes de los documentos consultados, los que permite una primera identificación de cuatro grandes tendencias en la cadena de suministro de la GISRS. Las tendencias se identifican a partir del contenido en cuanto a las palabras clave que forman parte de cada grupo de palabras, tal como se muestra en la Fig. 7. Hallazgos similares son los encontrado por Chen et al., (2017), que especifican que el campo de los Residuos de alimentos ha crecido más en los últimos 8 años.

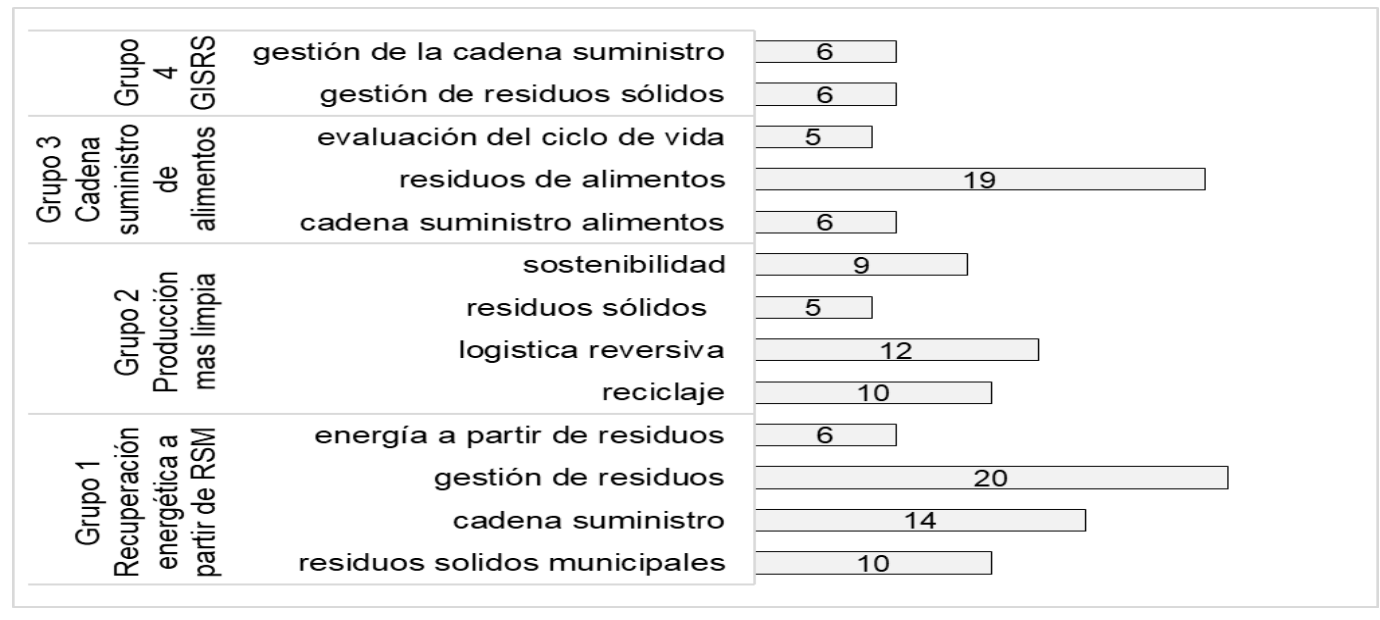

Fig. 7: Tendencias de investigación a partir de la literatura.

Los números indicados en cada barra, hacen alusión a la ocurrencia de cada término en la literatura consultada, es decir el número de veces que se menciona cada término en los documentos leídos. De acuerdo con este análisis de ocurrencias, puede decirse que el foco de atención está en la gestión de residuos sólidos (Caetano et al., 2017; Lohri et al., 2015) procedentes, en especial, de la cadena de suministro de la industria de alimentos (Campoy-Muñoz et al., 2017; Nagurney et al., 2012) mediante la combinación de acciones de mejoramiento como la logística reversiva (Bing et al. 2016) cuidando los indicadores de sostenibilidad (Ávila et al., 2017). Los grupos conformados en la Fig. 7, se establecen como grupos de oportunidades de trabajo para profundización a nivel de investigación aplicada, oportunidades que se denominan de aquí en adelante brechas. En la tabla 1 se relacionan estas cuatro brechas asociadas con herramientas de la ingeniería que pueden ser de apoyo para tratar aspectos relevantes con respecto a la sostenibilidad en las cadenas de suministro de la GISRS. A continuación, se describe el detalle de cada brecha como oportunidad de profundización, especificando experiencias y autores que han relacionado la importancia de tratar cada tema. 
Tabla 1: Herramientas de la ingeniería frente a cada brecha identificada.

\begin{tabular}{|l|l|}
\hline $\begin{array}{l}\text { Brechas } \\
\text { Brecha 1 - Recuperación energética a partir de RSM } \\
\text { Residuos sólidos municipales }\end{array}$ & $\begin{array}{l}\text { Herramientas de la Ingeniería } \\
\text { Métodos multicriterio } \\
\text { Factibilidad técnica, social, ambiental y económica }\end{array}$ \\
\hline $\begin{array}{l}\text { Cadenas de suministro } \\
\text { Energía a partir de residuos }\end{array}$ & Análisis del ciclo de vida \\
\hline $\begin{array}{l}\text { Brecha } 2 \text { - Producción más limpia } \\
\text { Reciclaje } \\
\text { Logística reversiva } \\
\text { Sostenibilidad }\end{array}$ & $\begin{array}{l}\text { Modelos de simulación } \\
\text { Modelos de optimización } \\
\text { Análisis del ciclo de vida }\end{array}$ \\
\hline $\begin{array}{l}\text { Brecha 3 - Gestión de la cadena de suministro de } \\
\text { alimentos. } \\
\text { Cadena suministro alimentos } \\
\text { Residuos de alimentos } \\
\text { Evaluación del ciclo de vida }\end{array}$ & $\begin{array}{l}\text { Métodos multicriterio } \\
\text { Análisis de ciclo de vida } \\
\text { Gestión Tecnológica }\end{array}$ \\
\hline $\begin{array}{l}\text { Brecha } 4 \text {-GISRS } \\
\text { Gestión de residuos sólidos de procesos } \\
\text { Gestión de cadena suministro }\end{array}$ & $\begin{array}{l}\text { Dinámica de sistemas } \\
\text { Modelos de simulación } \\
\text { Modelos de optimización } \\
\text { Análisis de ciclo de vida }\end{array}$ \\
\hline
\end{tabular}

Brecha 1: se establece a partir de conceptos como "waste to energy", "waste management", "supply chain" y "municipal solid waste"; este grupo de tópicos hace alusión al interés por gestionar los RSM a través del aprovechamiento energético (Nixon et. al., 2013a); para ese fin, es necesario estudiar y seleccionar opciones de aprovechamiento de RSM como un eslabón en la cadena de la GISRS (Steinvorth, 2014). Algunas de las herramientas de la ingeniería que pueden apoyar este tipo de decisiones son los métodos multicriterio y estudios de factibilidad técnica, ambiental, social y económica similares a la metodología de Yadav et. al., (2016) y la selección de fuentes de electricidad a partir de fuentes renovables para edificios comerciales como la aplicación de Restrepo-Garcés et al., (2016).

Brecha 2: con términos como "recycling", "reverse logistics", "solid waste" y "sustainability", puede inferirse el interés de las cadenas de suministro convencionales en interactuar con la GISRS a través de prácticas como la logística reversiva y el reciclaje para la gestión de sus residuos sólidos, con aplicaciones como las de Abbasi et al., (2010).Los modelos de optimización y simulación pueden ser de gran apoyo para este tipo de estudios (Dotoli et. al., 2006)

Brecha 3: donde se consideran conceptos como "food supply chain", "food waste" y "life cycle assessment" a través de los cuales se observa el interés de las cadenas de suministro de alimentos en incorporar herramientas de análisis industrial como el ciclo de vida de sus productos (Peças et. al., 2009). Las herramientas de gestión ambiental pueden complementarse con selección de alternativas de gestión de residuos (métodos multicriterio), herramientas de diseño industrial para nuevos productos (gestión tecnológica) y, finalmente herramientas para la identificación de procesos, componentes y sistemas, cuya contribución al impacto ambiental es significativa (Rahman y Coowanitwong, 2014).

Brecha 4: con conceptos como "solid waste management" y "supply chain management", que pueden verse como la integración de la gestión de las cadenas de suministro convencionales para aplicar dichas prácticas a la gestión de los residuos de una forma integral y sostenible (GISRS) bajo el modelo de Klunder y Anschütz, (2001). Herramientas de la ingeniería que pueden ser de apoyo para este fin, son igualmente los modelos matemáticos de optimización y simulación (Kovačić et. al., 2017), así como la dinámica de sistemas como lo ha trabajado Delle y Julin, (2013).

\section{DISCUSIÓN Y ANÁLISIS FINAL}

A través del análisis bibliométrico realizado, se recopilaron 298 documentos asociados a la recuperación de energía a partir de residuos sólidos para ser analizados en Bibexcel, Pajek y VOSviewer@ y así obtener agrupaciones por afinidad en cuanto a temas, autores y revistas, lo que permite inferir un acercamiento hacia la frontera de conocimiento de una o más áreas de investigación en los últimos 10 años. Los primeros autores más citados son Betz, Garrone y Girotto, quienes tienen un nivel de citación de 39, 54 y 49 respectivamente y, sus respectivos temas de investigación son Food waste management, Food waste valorization y Circular and Sustainable Bioeconomy; situación que deja ver desde primera instancia las tendencias y avances en materia de investigación. Las nacionalidades de los autores más destacados son los Estados Unidos con 28 
documentos asociados, seguido por Reino Unido con 25 documentos y finalmente el tercer país con más publicaciones de acuerdo con esta búsqueda es Brasil con 22 documentos.

Dentro del grupo de revistas que reportan artículos con referencia a Energía y Tecnología se destacan Waste Management con 390 documentos y su nivel de relación con otras revistas es de 93, lo que indica que comparte la temática de Energía y tecnología con 93 journals más. En el grupo de tecnologías medioambientales se destaca la revista Environmental Science \& Technology con 101 documentos y 42 conexiones con otras revistas y, finalmente, Journal of Cleaner Production que está dentro del grupo de Producción limpia tiene 317 documentos con un nivel de conexión de 50 revistas más.

En cuanto a las etapas de la GISRS que tienen mayor interés en la literatura corresponden al aprovechamiento de residuos con recuperación de energía y tecnologías limpias para la prevención en la generación de residuos sólidos con 18 y 10 documentos asociados respectivamente. En el detalle de las temáticas sobre WTE se destaca el eje de "Producción más limpia y mejoramiento" que reporta 20 autores que han basado sus investigaciones en este tema, seguido de "Cadena de suministro de alimentos" y "Recuperación energética a partir de residuos" con 17 autores trabajando en estos tópicos. Las brechas identificadas a partir de la literatura están enfocadas a ser temas de profundización en países en vía de desarrollo. Las herramientas de la ingeniería que se proponen constituyen un apoyo para facilitar la investigación aplicada en este tipo de países dado que en su mayoría son herramientas con un método definido y permiten evaluar los resultados.

\section{CONCLUSIONES}

A través del análisis bibliométrico realizado en este estudio se obtuvo un acercamiento hacia la frontera de conocimiento en el área de decisiones de tipo ambiental para el tratamiento de la fracción orgánica de los residuos sólidos urbanos, identificando una especial atención en el aprovechamiento de residuos con recuperación de energía y tecnologías limpias. Las brechas identificadas están enfocadas a ser temas de profundización en países en vía de desarrollo.

\section{REFERENCIAS}

Abbasi, M. N., C.Y. Wong y C. Lalwani, Recycling Attitudes and Behaviours: A Study of Plastics Recycling Supply Chains in Pakistan. doi.org/10.1049/cp.2010.0410. In 5th International Conference on Responsive Manufacturing - Green Manufacturing, ICRM 2010 (Vol. 2010, pp. 38-42). Institute of Management Sciences, Bahauddin Zakariya University, Multan, Pakistan (2010)

Ávila, C., K. Cedano y M. Martínez, Sustainability Analysis of Waste to Energy Strategies for Municipal Solid Waste Treatment, International Journal of Environmental Sustainability, 13(2) (2017)

Bing, X., J. M. Bloemhof, T.R.P. Ramos, A. P. Barbosa-Povoa, C.Y. Wong y J. G. A. J. Van der Vorst, Research Challenges in Municipal Solid Waste Logistics Management, http://doi.org/10.1016/j.wasman.2015.11.025, Waste Management, 48, 584-592 (2016)

Brancoli, P., K. Rousta y K. Bolton, Life Cycle Assessment of Supermarket Food Waste, Resources, Conservation and Recycling, 118, 39-46 (2017)

Caetano, M., D. Depizzol y A. De Oliveira Pereira Dos Reis, Analysis of Solid Waste Management and Improvement Proposal: A Case Study in Carpentry of Cariacica-ES, Gestao e Producao, 24(2), 382-394 (2017)

Calva-Alejo, C. L. y R. I. Rojas-Caldelas, Diagnóstico de la gestión de residuos sólidos urbanos en el municipio de Mexicali, México: Retos para el logro de una planeación sustentable. doi.org/10.4067/S0718-07642014000300009, Información Tecnologica, 25(3), 59-72 (2014)

Campoy-Muñoz, P., M. A. Cardenete y M.C. Delgado, Economic Impact Assessment of Food Waste Reduction on European Countries Through Social Accounting Matrices, doi.org/10.1016/j.resconrec.2017.02.010.Resources, Conservation and Recycling, 122, 202-209 (2017)

Chen, H., W. Jiang, Y. Yang, Y. Yang y X. Man, State of the Art on Food Waste Research: A Bibliometrics Study From 1997 to 2014.doi.org/10.1016/j.jclepro.2015.11.085, Journal of Cleaner Production, 140, 840-846 (2017)

Delle, J. S. y A. Julin. Energy Modeling For An-Eco-City with Focus on Biogas Potential. KTH School of Industrial Engineering and Management, Energy Technology EGI-2013-XX, Stockholm (2013)

Dotoli, M., M.P. Fanti, C. Meloni y M.C. Zhou, Design and Optimization of Integrated E-Supply Chain for Agile and Environmentally Conscious Manufacturing.doi.org/10.1109/TSMCA.2005.859189. IEEE Transactions on Systems, Man, and Cybernetics Part A: Systems and Humans, 36(1), 62-75 (2006)

Enrique, N., M. Gómez, M. Teresa, C. Galvis y E. Dugarte, Sostenibilidad Integral en la Cadena de Abastecimiento de la Producción de Biodiesel a Base de Aceite de Palma en Santander y Sur del Cesar, Encuentro Internacional de Educación En Ingeniería ACOFI. Colombia (2015) 
Fehr, M. y C.A. Arantes, Making a Case for Recycling Biodegradable Municipal Waste, Environment Systems and Decisions, 35(4), 483-489 (2015)

Fu, H.Z., Y.S. Ho, Y. M. Sui y Z.S. Li, A Bibliometric Analysis of Solid Waste Research During the Period 1993-2008, http://doi.org/10.1016/j.wasman.2010.06.008, Waste Management, 30(12), 2410-2417 (2010)

García-García, A., A. Pardo-lbáñez, A. Ferrer-Sapena, F. Peset y L. M. González-Moreno. Herramientas de Análisis de Datos Bibliográficos y Construcción de Mapas de Conocimiento: Bibexcel y Pajek. doi.org/https://dx.doi.org/10.1344/BiD2015.34.22. BiD: Textos Universitaris de Biblioteconomia i Documentació, 34(34), 8 (2015)

Hoornweg, D., P. Bhada-Tata, What a Waste: A Global Review of Solid Waste Management.doi.org/10.1111/febs. 13058.The FEBS Journal, 281(19), 4556. Banco Mundial, Estados Unidos de América, Washington (2012)

Huang, I. B., J. Keisler e I. Linkovl, Multi-Criteria Decision Analysis in Environmental Sciences: Ten Years of Applications and Trends. doi.org/10.1016/j.scitotenv.2011.06.022, The Science of the Total Environment, 409(19), 3578-94 (2011)

Hupponen, M., K. Grönman y M. Horttanainen, How Should Greenhouse Gas Emissions be taken into Account in The Decision Making of Municipal Solid Waste Management Procurements? A case Study of the South Karelia Region, Finland. http://doi.org/10.1016/j.wasman.2015.03.040, Waste Management, 42, 196-207 (2015)

Klunder, A. y J. Anschütz, Integrated Sustainable Waste Management - the Concept. Tools for Decision-makers. Experiences from the Urban Waste Expertise Programme (1995-2001). 44 p.: 3 fig., 6 photogr., 1 tab. Publisher WASTE. ISBN Number 9076639027, The Netherlands. Gouda (2001)

Kovačić, D., J. Usenik y M. Bogataj, Optimal Decisions on Investments in Urban Energy Cogeneration Plants - Extended MRP and Fuzzy Approach to the Stochastic Systems.doi.org/10.1016/j.ijpe.2016.02.016, International Journal of Production Economics, 183, 583-595 (2017)

Lohri, C. R., A. Faraji, E. Ephata, H.M. Rajabu y C. Zurbrügg. Urban Biowaste for Solid Fuel Production: Waste Suitability Assessment and Experimental Carbonization in Dar Es Salaam, Tanzania. doi.org/10.1177/0734242X14564644, Waste Management and Research, 33(2), 175-182 (2015)

López, A., D. Méndez, A. Paz y H. Arboleda, Desarrollo e Instrumentación de un Proceso de Vigilancia Tecnológica basado en Protocolos de Revisión Sistemática de la Literatura, doi.org/10.4067/S0718-07642016000400017, Información Tecnológica, 27(4), 155-164 (2016)

Meadows, J., D. Coote y M. Brown, The Potential Supply of Biomass for Energy from Hardwood Plantations in the Sunshine Coast Council Region of South-East Queensland, Australia, Small-Scale Forestry, 461-481 (2014)

Nagurney, A., A.H. Masoumi y M. Yu, Supply Chain Network Operations Management of a Blood Banking System with Cost and Risk Minimization.doi.org/10.1007/s10287-011-0133-z, Computational Management Science, 9(2), 205-231 (2012)

Nixon, J. D., P. K. Dey, S. K. Ghosh y P. A. Davies, Evaluation of Options for Energy Recovery from Municipal Solid Waste in India Using the Hierarchical Analytical Network Process. doi.org/10.1016/j.energy.2013.06.052, Energy, 59, 215-223 (2013)

OXFAM Intermón, Definición de Sostenibilidad: ¿Sabes Qué Es y Sobre Qué Trata? | Ingredientes que Suman (2018)

Peças, P., I. Ribeiro, R. Folgado y E.A. Henriques, A Life Cycle Engineering Model for Technology Selection: a Case Study on Plastic Injection Moulds for Low Production Volumes, doi.org/10.1016/j.jclepro.2009.01.001, Journal of Cleaner Production, 17, 846-856 (2009)

Persson, O., R. Danell y J. Wiborg Schneider. How to use Bibexcel for Various Types of Bibliometric Analysis. In Celebrating Scholarly Communication Studies: a Festschrift for Olle Persson at his 60th Birthday, ed. F. Åström, R. Danell, B. Larsen, J. Schneider, 9-24. Leuven, Belgium: International Society for Scientometrics and Informetrics (2009)

Rahman, M. y N. Coowanitwong Better City Environment Through ISO 14001 Applications and Strategic Decision-Making Process. In 6th International Conference on Environmental Informatics, ISEIS 2007, International Society for Environmental Information Sciences (2014)

Restrepo-Garcés, A.R., D.F. Manotas-Duque y C. A. Lozano, Portafolio para Auto-Generación de Electricidad con Fuentes Renovables en Edificios Comerciales doi.org/10.4067/S0718-07642016000100011, Información Tecnologica, 27(1), 91$104(2016)$

Steinvorth, A., Aprovechamiento Energético de Residuos Sólidos Municipales Mediante el Uso de Tratamientos Térmicos de Avanzada, Éxito Empresarial, CEGESTI, 253 (2014)

Tchobanoglous, G., y F. Kreith. Handbook of Solid Waste Management, $2^{\text {a }}$ Ed., McGraw-Hill Professional. Estados Unidos de América (2002)

Van Eck, N. y L. Waltman. VOSviewer: A Computer Program for Bibliometric Mapping. Copyright (C) 2018 Centre for Science and Technology Studies, Leiden University. The Netherlands (2018)

Yadav, V., S. Karmakar, A. K. Dikshit y S.A. Vanjari. A Feasibility Study for the Locations of Waste Transfer Stations in Urban Centers: A Case Study on the City of Nashik, India. doi.org/10.1016/j.jclepro.2016.03.017. Journal of Cleaner Production (2016) 